\title{
SOLUTE SEGREGATION AND AGGREGATION IN Mg ALLOYS
}

\author{
J.F. Nie ${ }^{1}$, Y.M. Zhu ${ }^{1}$, N.C. Wilson ${ }^{2}$ \\ ${ }^{1}$ Department of Materials Engineering, Monash University; Victoria 3800, Australia \\ ${ }^{2}$ CSIRO Manufacturing Flagship; Clayton, Victoria 3168, Australia
}

Keywords: Magnesium alloys, Solute segregation, Solute aggregation, Microstructures

\section{Extended Abstract}

The addition of adequate amounts of rare-earth (RE) alloying elements can produce appreciable effects on microstructures, formability and mechanical properties of magnesium alloys. At low concentrations, it can weaken the basal texture in the asdeformed and the recrystallized conditions in wrought alloys and improve the creep resistance of casting alloys at elevated temperatures; at high concentrations, it can bring significant age hardening effects and thus improve the alloy strength. While this knowledge has been known for some years, the precise roles of $\mathrm{RE}$ solutes in texture weakening and the early stage of precipitation remain unclear.

It has been demonstrated in recent years that the application of high-angle annular dark-field scanning transmission electron microscopy (HAADF-STEM) can reveal the distribution of RE solutes and the structure of precipitate phases at the atomic scale. The use of this Z-contrast imaging technique, combined with atom probe tomography or other characterization techniques such as energy dispersive X-ray spectroscopy and electron energy loss spectroscopy, has revealed the occurrence of solute segregation in high-angle grain boundaries even in solid solution single phase alloys based on the Mg-Gd system [1-4]. The segregation of RE atoms in the grain boundaries will no doubt impede the boundary migration [5] and thus change the recrystallization behaviours and perhaps even the recrystallization texture. In addition to the wellestablished solute segregation in the partially-coherent high-angle grain boundaries, a very recent study using HAADF-STEM [6] has revealed the occurrence of a periodic segregation of solute atoms in fully-coherent twin boundaries of $\{10 \overline{1} 1\}_{\alpha},\{10 \overline{1} 2\}_{\alpha}$ and $\{10 \overline{1} 3\}_{\alpha}$. This segregation results from the minimization of elastic strain energies associated with twin boundaries and the over-sized individual solute atoms, and it provides a strong pinning of the twin boundaries and a concomitant annealing hardening effect when the alloy is deformed under certain circumstances. Subsequent studies using first-principles density functional theory computations indicate that solute atoms of most commonly used alloying elements have a strong tendency to segregate to $\{10 \overline{1} 2\}_{\alpha}$ twin boundaries $[7,8]$, and that solute segregation can also occur in a symmetrical tilt boundary [9].

In concentrated Mg-RE alloys, the intermediate precipitate phases such as $\beta^{\prime}$ and $\beta_{1}$ have been relatively well characterized in recent years [10]. However, the structures and compositions of precipitate phases, GP zones and clusters that form in the early stage of decomposition are still poorly understood [11,12]. It remains to be firmly established whether solute clusters form and, if so, how they interact with lattice defects such as dislocations and twins. Such information is critically needed if some of the beneficial and yet mysterious effects of RE additions are to be rationalized. This presentation will also provide a review of our recent findings in the study of solute aggregates in a group of magnesium alloys using HAADF-STEM and first-principles density functional theory.

\section{References}

[1] M. Bugnet, A. Kula, M. Niewczas, and G.A. Botton, "Segregation and Clustering of Solutes at Grain Boundaries in Mg-Rare Earth Solid Solutions," Acta Mater. 79 (2014), 66-73.

[2] J.P. Hadorn, T.T. Sasaki, T. Nakata, T. Ohkubo, S. Kamado, and K. Hono, "Solute Slustering and Grain Boundary Segregation in Extruded Dilute Mg-Gd Alloys," Scripta Mater. (2014) in press.

[3] N. Stanford, G. Sha, J.H. Xia, S.P. Ringer, and M.R. Barnett, "Solute Segregation and Texture Modification in An Extruded Magnesium Alloy Containing Gadolinium," Scripta Mater. 65 (2011), 919-921.

[4] J.P. Hadorn, K. Hantzsche, S.B. Yi, J. Bohlen, D. Letzig, J.A. Wollmershauser, and S.R. Agnew, "Role of Solute in the Texture Modification during Hot Deformation of $\mathrm{Mg}$ Rare Earth Alloys," Metall. Mater. Trans. A 43A (2012), 1347-1362.

[5] J.D. Robson, "Effect of Rare-Earth Additions on the Texture of Wrought Magnesium Alloys: The Role of Grain Boundary Segregation," Metall. Mater. Trans. A 45A (2014), 3205-3212.

[6] J.F. Nie, Y.M. Zhu, J.Z. Liu, and X.Y. Fang, "Periodic Segregation of Solute Atoms in Fully Coherent Twin Boundaries," Science, 340 (2013), 957-960.

[7] J. Zhang, Y. Dou, and Y. Zheng, "Twin-Boundary Segregation Energies and Solute-Diffusion Activation Enthalpies in Mg-Based Binary Systems: A First-Principles Study," Scripta Mater. 80 (2014), 17-20.

[8] M. Ghazisaeidi, L.G. Hector Jr, and W.A. Curtin, "Solute Strengthening of Twinning Dislocations in Mg Alloys," Acta Mater. 80 (2014), 278-287.

[9] L. Huber, J. Rottler, and M. Militzer, "Atomistic Simulations of the Interaction of Alloying Elements with Grain Boundaries in Mg," Acta Mater. 80 (2014), 194-204.

[10] J.F. Nie, "Precipitation and Hardening in Magnesium Alloys," Metall. Mater. Trans. A 43A (2012), 3891-3939.

[11] W. Lefebvre, V. Kopp, and C. Pareige, "Nano-Precipitates Made of Atomic Pillars Revealed by Single Atom Detection in a Mg-Nd Alloy," Appl. Phys. Lett. 100 (2012), 141906.

[12] Y. Matsuoka, K. Matsuda, K. Watanabe, J. Nakamura, W. Lefebvre, D. Nakagawa, S. Saikawa, and S. Ikeno, "Precipitation Sequence in the Mg-Gd-Y System Investogated by HRTEM and HAADF-STEM," Mater. Trans. 55 (2014), 1051-1057. 